\title{
Construir la identidad del inspector de zona escolar en tiempos de complejidad
}

\author{
Constructing the Identity of the School Zone Inspector in Times of \\ Complexity
}

\section{Construindo a identidade do fiscal da zona escolar em tempos de complexidade}

\author{
Raquel Susana Sandrone* (iD \\ *Doctoranda en Educación. Licenciada en EGB. Actualmente Inspectora de Zona \\ Escolar 2350- Balnearia Córdoba. https://orcid.org/0000-0001-6572-6409
}

\section{OPEN ACCESS \\ DOI: http://dx.doi.org/10.18634/sophiaj. 17v.1i.1053}

Información del artículo

Recibido: octubre 8 de 2020

Revisado: enero 10 de 2021

Aceptado: marzo 15 de 2021

Publicado: Abril 20 de 2021

Palabras clave: Inspector de zona, calidad educativa, enseñanza, educación, aprendizaje

Keywords: Zone inspector, educational quality, teaching, education, learning.

Palavras-chave: Inspetor de zona, qualidade educacional, ensino, educação, aprendizagem.

\section{Cómo citar: /how cite:}

Sandrone, R.S (2021) Construir la identidad del inspector de zona escolar en tiempos de complejidad. Sophia 17 (1), e1053

Sophia-Educación, volumen 17 número 1. enero/junio 2021. Versión español

\section{RESUMEN}

Inmersos en el siglo XXI, caracterizado por el fin de las certezas, es necesario transmutar las formas de hacer escuela. Dentro del sistema educativo, la institución supervisión escolar cumple un papel fundamental en estas nuevas formas de ser y estar dentro de las escuelas. Es en su función pedagógica y desde el rol de garante de la formación continua de todos los docentes de su jurisdicción que accederá a la mejora esperada. El ensayo consiste en la presentación de los antecedentes más relevantes del Proyecto de Tesis Doctoral, elaborado en el marco del Doctorado en Educación de la Universidad Católica de Córdoba. El objetivo es explorar y describir la trayectoria de la función formadora del inspector de zona. Se busca explicar sobre la tendencia que va adoptando en el transcurso del tiempo, considerando que el aporte académico impactaría en la desnaturalización de la función formadora del supervisor, tan necesaria para dar respuestas a la realidad del presente siglo: acompañar a los directivos y docentes para que la reflexividad sea su práctica orientada al mejoramiento educativo en respuesta a la incertidumbre que caracteriza la contemporaneidad. La idea es construir la identidad del inspector de zona escolar en la actualidad, desde diferentes perspectivas: teórica, práctica, histórica y jurídica. 
Copyright 2021 Universidad La Gran Colombia

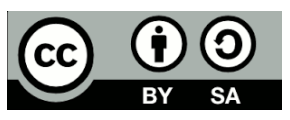

Conflicto de interés:

Los autores declaran no tener ningún conflicto de interés.

Correspondencia de autor:

rasandrone67@gmail.com

\section{ABstRact}

Immersed in the 21st century, characterized by the end of certainties, it is necessary to transmute the ways of doing school. Within the educational system, the institution of school supervision plays a fundamental role in these new ways of being and being within schools. It is in its pedagogical function and from its role as guarantor of the continuous training of all teachers in its jurisdiction that it will achieve the expected improvement. The essay consists of the presentation of the most relevant background of the Doctoral Thesis Project, elaborated within the framework of the Doctorate in Education of the Catholic University of Córdoba. The objective is to explore and describe the trajectory of the training function of the zone inspector. The aim is to explain the tendency adopted over time, considering that the academic contribution would have an impact on the denaturalization of the supervisor's training function, so necessary to give answers to the reality of the present century: to accompany managers and teachers so that reflexivity becomes their practice oriented to educational improvement in response to the uncertainty that characterizes contemporaneity. The idea is to build the identity of the school zone inspector today, from different perspectives: theoretical, practical, historical and legal.

RESUMO

Imersos no século 21, marcado pelo fim das certezas, é preciso transmutar os modos de fazer escola. No sistema educacional, a instituição de supervisão escolar desempenha um papel fundamental nessas novas formas de ser e estar dentro da escola. É na sua função pedagógica e a partir do papel de garante da formação contínua de todos os docentes da sua jurisdição que terá acesso à melhoria esperada. 0 ensaio consiste na apresentação dos antecedentes mais relevantes do Projeto de Tese de Doutorado, elaborado no âmbito do Doutorado em Educação da Universidade Católica de Córdoba. O objetivo é explorar e descrever a trajetória da função de treinamento do inspetor de zona. Busca explicar a tendência que vem adotando ao longo do tempo, considerando que a contribuição acadêmica impactaria na desnaturalização da função formativa do supervisor, tão necessária para responder à realidade deste século: acompanhar gestores e professores para que a reflexividade seja sua prática almejada na melhoria educacional em resposta à incerteza que caracteriza a contemporaneidade. A ideia é construir a identidade do atual inspetor da zona escolar, a partir de diferentes perspetivas: teórica, prática, histórica e jurídica. 


\section{Introducción}

Esta investigación nació con el propósito de sostener las prácticas de los inspectores de zona en la realidad compleja en que interactúan, ya que es escaso el bagaje académico que marque los "cómo" de la función formadora del supervisor. Motiva la misma la situación personal y profesional del investigador, quien se desempeña como inspector de zona en el sistema educativo, por lo que busca constantemente la mejora de sus propias prácticas.

Mucho se ha preguntado y respondido acerca del inspector/supervisor en diferentes lugares y desde variadas facetas, pero hasta donde se ha podido indagar, no existe un estudio riguroso que sistematice los modos en que se lleva a cabo la función formadora del inspector de zona. Se adopta este nombre dado que es la denominación del cargo en el contexto donde se aplica la investigación.

La literatura académica instala un discurso que interviene el rol del inspector de zona desde diferentes ópticas que permiten ampliar y complementar la mirada formada por el investigador desde los datos analizados. Es a través de la investigación documental que se avanza en la construcción de la identidad del inspector de zona escolar.

\section{Desarrollo del tema}

\section{La visión de varios autores}

El inspector de zona escolar en el siglo XXI encuentra una nueva identidad íntimamente relacionada con la mejora de la calidad de la enseñanza y los aprendizajes desde una mirada innovadora. Todos los autores, con los cuales se adhiere, lo definen por las funciones que desarrolla dentro del sistema. Motivo por el cual, muchos lo nominan supervisor, ya que, el paso del tiempo le imprimió un significativo cambio de roles.

En la literatura más actualizada varios autores hablan de esa dualidad de funciones: Podestá en Gvirtz y Podestá (2012) recupera de Blejmar (2005), un interrogante: “¿inspección o servicio?”, atribuyéndole la responsabilidad de ser un actor clave, pero que la toma de decisiones puede convertirse en una amenaza o en una oportunidad. También Aguerrondo en Gvirtz y Podestá (2012) reconoce la tensión entre asistencia y control y afirma que la tarea base del nuevo supervisor es trabajar para la articulación, para conformar un único sistema educativo flexible y capaz de adaptarse a diferentes situaciones.

Podestá en Gvirtz y Podestá (2012) habla del supervisor como el catalizador del sistema educativo, el que contribuye a acelerar el cambio y el fortalecimiento institucional en las escuelas. Lo cataloga de dinamizador de la tarea hacia el interior de las instituciones. Y en esa misma línea, Gvirtz-Abregú en Gvirtz y Podestá (2012) lo designan como facilitador organizacional.

Rivas y Furman (2017) lo definen como la bisagra entre las políticas y las escuelas. Se considera una bisagra entre lo macro y lo micro del sistema, pues como agente del estado, es conocedor de la política educativa la cual actualiza a su territorio, garantizando así el carácter sistémico de la educación. Así mismo, es agente del sistema porque comprende los contextos en los que se insertan sus escuelas.

Es también agente conservador, encargado del orden y el control, quien garantiza los derechos por vía de la continuidad, de lo común y de la normativa. Y paradójicamente, también es agente de cambio, de movilización, de replanteo, de transformación. Un agente atado a la realidad de su zona, el hacedor en la resolución de problemas y a la vez, un agente estratégico, con visión sistémica.

Nicastro (2019) sostiene que el supervisor es un representante del Estado, un funcionario público del sistema educativo, un representante de estas políticas, garante del desarrollo de estas en las situaciones concretas que le competen en el marco de sus zonas. En consecuencia, cualquier norma o regulación, opera como una prescripción que da vida a las escuelas, pero que no son simples aplicaciones. Pues el rol experto del inspector, va ajustando, creando, para que no sea simple ejecución. Pero a la vez es necesario generar dispositivos de acompañamiento que también permiten modalidades de vigilar, es decir, de garantizar el desarrollo de un marco normativo. 
Casal, V. (2019) lo simplifica en "es allí donde la macropolítica hace "zoom" (p.1) sobre las salas y las aulas". Entonces, ante la pregunta ¿quién es el inspector de zona escolar? Un intento de respuesta simplificada podría referirse a:

Es el representante del estado en un determinado sector del sistema, encargado de garantizar a través de su intervención, la materialización de la política pública en las instituciones educativas bajo su jurisdicción. Es también un agente estratégico y sistémico, con mirada holística y pensamiento prospectivo, gestor del cambio para la mejora de las condiciones de enseñanza y el aprendizaje. Conoce la realidad particular de cada contexto, trasmite a los niveles más alto en la jerarquía los desafíos que surgen de dichas situaciones y actúa en consecuencia a favor de las trayectorias escolares de todos los estudiantes.

La complejidad en la que gestiona el inspector de zona, permite complementar el concepto desde diferentes aristas. Algunas de ellas son abordadas a continuación.

\section{El inspector de zona escolar en distintos tiempos y espacios}

Conocer quién es el inspector de zona en el presente, necesita una mirada al pasado, a su origen, y también a la evolución que tuvo a lo largo del tiempo. Con esta perspectiva, se bucea en los estudios existentes sobre el tema en búsqueda de los procesos de su constitución histórica.

A fin de atesorar las tradiciones y propagar los valores atesorados por la sociedad del momento, surge la supervisión educativa como control político de vigilancia de las escuelas y los maestros. Por su parte, la epistemología -ciencia que trata la definición del conocimiento y de los conceptos afines con las fuentes, los criterios, los tipos de saberes posibles y el grado con el que resulta cierto cada uno; además de la relación entre el sujeto que conoce y el objeto conocido- permite entender su origen. Y el caso particular de la Epistemología de la Educación, lleva al estudio del conocimiento del conocimiento (Morin,1994). Particularmente, cuando hablamos de Supervisión Educativa, la epistemología nos acerca al estudio de su esencia, su práctica, sus formas de gestión y actuación en las escuelas de su jurisdicción.

Los primeros datos que históricamente se encuentran sobre el rol del supervisor datan de la Edad Antigua. El sofronista se denominaba en Atenas y era un magistrado con la función de vigilar la educación y la conducta moral. El areópago era parte del tribunal superior y su función era la de controlar en la escuela la acción pública. Los éforos, magistrados elegidos por el pueblo en Esparta, también cumplían el rol de controlar la educación pública y en Roma estaban los censores que eran quienes tenían el control y autoridad absoluta de la educación de los jóvenes, todos ellos magistrados facultados por la ley.

Durante la Edad Media, fueron los claustros y monasterios los encargados de las escuelas en las civilizaciones de occidente. La Iglesia era la encargada de dirigir e inspeccionar todas las instituciones educativas existentes. El gran chantre era el nombre que recibía en España. Maestrescuela es el nombre que, en el siglo XII, recibían los que se dedicaban a la inspección escolar.

Por su parte, en la Edad Moderna, la justicia era quien se hacía cargo en Europa de la inspección de la enseñanza. Al mismo tiempo que el Estado Moderno cimienta su función administrativa, la inspección de educación aparece con la base del sistema educativo.

En Argentina, a fines del siglo XIX, la inspección escolar aparece y acompaña el comienzo de los sistemas educativos. La escuela se configuró como un modo de regulación social de los sujetos, marcando mecanismos de autodisciplina, formando una estructura cognitiva con esquemas de clasificación y una serie de normativas que definían lo que era aceptable, normal, posible. El carácter verticalista y la promoción desde las instancias centrales del Estado admitieron la presencia del sistema de inspección como control de la norma.

Algunos autores encontrados dan cuenta del proceso histórico de los mismos. Por un lado, Aguerrondo (2012) habla de tres etapas históricas y de evolución donde se organiza la función del Inspector. Habla de una primera etapa que comprende hasta mediados del siglo XX y que la llama de inspección; una segunda etapa que se extiende hasta fines del siglo XX, la de supervisión y, por último, en el siglo XXI, la de facilitador/auditor. 
Por su parte, Senestrari (2015) desarrolla una construcción cronológica de su evolución. Según un informe de la DiNIECE (2013), históricamente, a partir de 1880, la inspección de escuelas se constituyó como un trabajo dependiente del Estado.

La primera etapa, o etapa de inspección, hace referencia a la función del supervisor como mediador entre la macro y la micropolítica, o sea entre el ministerio y las escuelas, pero desde el control de las mismas. Es una mirada totalmente aplicacionista de las normas y regularidades dictadas por el poder político y que posiciona al inspector como responsable de su funcionamiento en las prácticas. Su inicio se remonta al siglo XIX, según dichos de Puiggros y citados por Senestrari (2015), cuando se organiza un marco legal en Argentina para la educación elemental. Así, las normas de esta época de fundación del sistema educativo conceden a las inspecciones de escuela las siguientes funciones: planificar, decidir, ejecutar y controlar el cumplimiento de la política educativa.

Es el representante del estado y su función se focalizó en definir los principios y las regulaciones sobre la organización y la metodología de la enseñanza en el aula. Sobre todo, la habilitación de los docentes era un común denominador, ya que la mayoría no conocían los programas, la enseñanza ni el uso del material didáctico. El control del cumplimiento del mandato pedagógico era la lógica de acción. El inspector tenía un trabajo en solitario, su tarea consistía en visitar las escuelas para visar su funcionamiento pedagógico, dejando registro de su paso en una memoria evaluativa con directivas de acción. Su autoridad era indiscutida, su visita un hecho excepcional e importante. El inspector ascendía a su cargo por mandato superior donde le valía la experiencia y la idiosincrasia.

El normalismo exigía la homogeneidad del sistema, la pedagogía positivista no reconocía la identidad social de la población presente en las aulas. La política de la época se reducía a la formación en la moral laica, nacionalista, liberal y universalista caracterizada por una lógica que no necesitaba ser demostrada, el método riguroso defendido por el Círculo de Viena: El inspector era el encargado del control del sistema educativo.

Southwell (2015), expresa que la bases que formaron el campo pedagógico del saber fueron la sanción de la ley de subvenciones nacionales a las provincias por un lado y por el otro, el proceso de creación de las escuelas normales. Al mismo tiempo se produjo el desarrollo de un campo del saber burocrático. Consecuentemente, el campo pedagógico generó un constructo de prescripciones sobre el aula mientras que el campo burocrático produjo la aparición de los inspectores del Consejo Nacional de Educación, los cuales, se encargaron en los sistemas provinciales de elaborar las reglas. El marco legar se consolidó entre las décadas de 1870 y 1890 para la educación elemental, las cuales fueron la base del sistema educativo.

La sanción de la Ley de Subvenciones Nacionales N463 (1871), permite el financiamiento nacional a las provincias a fin de administrar la instrucción pública y a la vez, establece la creación de comisiones provinciales con la finalidad de indicar cuáles son las funciones de los inspectores nacionales en el territorio provincial que dependan de estas comisiones.

En su libro Educación Popular, Sarmiento (1849) ocupa todo un capítulo dirigido a los inspectores. El mismo lleva el nombre de "Inspección de las Escuelas Públicas".

La Ley de Educación Común de la provincia de Buenos Aires (1875) fue el principal antecedente para la sanción de la Ley de Educación Común, N¹420 (1884) de carácter nacional. El desarrollo del Consejo Nacional de Educación y la tarea de los inspectores fueron hechos paralelos y produjeron los debates que enmarcaron la Ley 1420. Uno de esos debates tiene que ver con el avance cuantitativo de la escolarización, que regula a las características de las escuelas, a la formación de los maestros y a los métodos de métodos utilizados como medios de calidad en educación. Esto produjo una mirada más pedagógica de los Inspectores del momento.

La expansión de la educación fue impulsada por un conjunto de políticas cuyo lugar central de gestión lo tuvo el Consejo Nacional de Educación. Entre ellas se puede mencionar: la creación de escuelas, la prescripción de metodología y enseñanzas que para ese entonces eran consideradas las más validas, la entrega de títulos y certificados y políticas destinadas a la inspección. Los inspectores fueron una de las figuras claves en esta formación del gobierno escolar desde un lugar intermedio entre la política de gobierno en incipiente expansión y la micropolítica de las instituciones educativas. 
Es de considerar que, a fines del siglo XX, la idea de inspección como control sufre importantes cambios que se vinculan con la expansión y el desarrollo de los diferentes niveles y modalidades, con la pedagogía del momento y sus debates y con las diferentes normativas que conformaron el sistema educativo en los diferentes países.

Se observa así, que la Ley 1420 (1884) promueve como función del inspector de escuela primarias la de controlar, vigilar, fiscalizar y garantizar el buen funcionamiento del servicio educativo bajo su jurisdicción. Pero en cambio, el Estatuto del Docente $(1958)^{1}$, y dando respuesta a los cambios que en el país y en otros países se venían produciendo en torno a la función de asesoramiento como la función primordial del supervisor y la de control y coordinación como funciones secundarias.

Varias investigaciones analizan la historia del supervisor en Argentina y expresan que las diferentes normas definieron, en el proceso de configuración del sistema educativo, a las funciones del supervisor educativo la asignación de tareas de evaluación y control, como también así, de asesoramiento y orientación. No obstante, la centralización del sistema educativo de Argentina, favoreció a las tareas de la supervisión priorizando las de control que cobran legitimidad en la posición de jerarquía que ocupa el inspector dentro de la organización en forma piramidal. Así, esta posición presenta al inspector con una figura que se vincula al cumplimiento de las normas que emana el Consejo Nacional de Educación.

De esta manera, en todo el territorio nacional, el inspector, funcionario del Estado, tenía como tarea fundamental y central el control del espacio público de la educación. Estos inspectores tuvieron un destacado papel en esos tiempos en los debates políticos y pedagógicos y en la definición de las orientaciones que formaron el sistema, plasmada en los planes de estudio, en las reglamentaciones y en la evaluación de los libros de textos. Algunos de esos hombres ocuparon cargos políticos muy destacados. Entre ellos podemos mencionar a Varela, Sobral, Lugones, Alcorta, entre otros.

Desde la conformación del Sistema Educativo Argentino, Córdoba contaba con instituciones educativas nacionales que se regían por la normativa de su jurisdicción y en las escuelas de la provincia estaban los consejeros escolares de distrito que eran las comisiones inspectoras que tenían la función de cuidar la higiene y moralidad en las escuelas particulares y públicas de la jurisdicción, proponer el equipo de gestión, recaudar, entre otras.

Con la Ley N¹426 Ley orgánica de educación primaria de la provincia de Córdoba (1896), se legisla sobre la función de la inspección. En el capítulo III artículo 26 se define que la administración local y el gobierno inmediato de las escuelas estaría a cargo de inspectores de sección y comisiones locales. Cabe recordar que en ese capítulo también se define la existencia de la dirección y administración a cargo de la Dirección General de Escuelas, hoy Dirección General de Educación Primaria.

El director general de escuelas de la provincia, de acuerdo con lo que dictaba el artículo 40 inc. 7 de la ley mencionada en el párrafo anterior, definía su rol a partir de ordenar la inspección general a fin de que los inspectores seccionales hicieran sus giras.

En el capítulo VI art. 42 se define que "la inspección de las escuelas primarias de la provincia se efectuará por medio de un inspector general e inspectores de sección en número suficiente que se fijara en el presupuesto anual y los nombramientos estarán a cargo del poder ejecutivo provincial.

La segunda etapa, o etapa de supervisión, tiene lugar aproximadamente en los años sesenta, cuando en América se produjeron las primeras reformas educativas. El contexto epocal ha cambiado, la industria y la migración del campo a la ciudad, produjo cambios en la sociedad y la masificación de la escuela. El sistema educativo se fragmenta y responde de distinta manera a los diferentes sectores sociales.

Para los supervisores, que siguen teniendo un trabajo en solitario, se habilitan las oficinas administrativas gubernamentales por el aumento de escuelas que es insostenible para el número de inspectores en plantel. Tampoco el presupuesto es acorde con la posibilidad de viajar a cada escuela constantemente. El supervisor

1. Ley 14.473. Estatuto del docente. Sancionada el 12 de septiembre de 1958 y publicada en el Boletín Oficial el 27 de septiembre de 1958. Extraída de http://www. bnm.me.gov.ar/giga1/normas/8178.pdf 
se torna, en consecuencia, en un agente con trabajo de carácter más administrativo donde los expedientes ocupan su tiempo y sus intervenciones.

Es esta una época en que se ponen de moda las planificaciones por lo que también, la tarea del inspector es la de realizar diagnósticos institucionales. Las visitas a las escuelas en este período se focalizan en el control de lo administrativo y de la planificación.

En Córdoba se legisla y sanciona el Estatuto Docente (1957) definiéndose el escalafón, los ascensos, la carrera, los deberes y las funciones. El inspector general "asiste a su despacho y atiende los asuntos de los inspectores de sección, dirige y vigila los trabajos de los inspectores de sección para que se cumpla con los decretos, reglamentos escolares y disposiciones del consejo general de escuelas".

El inspector de sección, hoy llamado zonal, tenía la función de vigilar la enseñanza, corregir los errores o defectos de las prácticas de la enseñanza, controlar los registros estadísticos, matrículas, de acuerdo con las disposiciones de la dirección o inspección general. El rol se orientaba también a dar conferencias pedagógicas y populares, promover la creación y fomento de bibliotecas populares, museos pedagógicos, entre otros.

Esta normativa define el acceso por concurso de oposición a los cargos directivos y de inspección. Además, el estatuto docente menciona en varios párrafos a los inspectores, otorgándoles la función de garante y fiscalizador de los derechos de los docentes.

Cabe agregar que en los años 70 aparecen planteamientos al nombre de inspección/inspector ya que tiene una gran connotación a la función de control asociada a la palabra inspección. Para dar respuesta a dichos cuestionamientos, se comienza a utilizar el nombre de supervisor/supervisión, que da cuenta de la preponderancia que empezaba a brindarse a la función del inspector desde la mirada de modelos más modernos y que tenían que ver con las tareas de asesoramiento por encima de las de control.

Es aquí donde notamos el primer cambio epistemológico en la Supervisión, que concuerda con el posicionamiento de Kant que por primera vez en la historia de la filosofía, demuestra que el objeto no es una cosa ajena al sujeto, algo externo y opuesto a éste. La función de la objetividad, según Kant, es una forma de la actividad del sujeto, y el propio sujeto no existe fuera de las cosas conocidas por él. Además, según el filósofo alemán, el objeto sólo existe en las formas de la actividad subjetiva y sólo así puede ser conocido. La cosa en sí, es decir, la realidad existente fuera de cualquier relación con el sujeto cognoscente es dada al sujeto solamente en la forma de los objetos. Los objetos son en su esencia producto de la actividad creadora propia del sujeto.

El análisis de Veleda (2011) caracteriza al inspector como "compensador dual” (p.29). Los aportes de la política educativa disponen de modalidades de compensación distributivas, como lo son los comedores escolares, la entrega de equipamientos y el financiamiento a las escuelas de sectores privados de derechos. Se busca la igualdad a través de programas sociales, pero se continúa con el modelo de enseñanza tradicional, el currículum graduado y la enseñanza simultánea, la presencialidad y la meritocracia. En estos escenarios el supervisor se enmarcó en procesos sociales del contexto de la dictadura, volviéndose autoritario, y de la democratización de las instituciones donde adopta un papel más humanizado. La convivencia y la formación en ciudadanía eran su norte.

La masividad que marcaba el rumbo de las escuelas es agravada por otro factor, la diversidad de modelos educativos que provoca no solo que los puestos docentes se diversifiquen, sino que ocurra lo mismo con los inspectores ya que son requeridos para nuevas modalidades de enseñanza. Frente al aumento en la complejidad del sistema, se produce un aumento en la cantidad de inspectores. Aparecen las inspecciones específicas como la de educación física, artística, entre otras.

En Córdoba, a fines del siglo XX, comienza una serie de reformas para la vuelta a la democracia, entre ellas, la Constitución, la ley educativa, los lineamientos curriculares, entre otros. La reforma educativa aparece para mejorar la calidad y ampliar los derechos a la educación. La misma propone un rol del supervisor de carácter democrático, participativo y regionalizado. 
La Resolución 1892/83 de Córdoba se completa en el noventa y uno con la Ley 8114, Ley General de Educación de la Provincia de Córdoba (1991) que otorga a los inspectores funciones diferentes a las tradicionales y en el capítulo II habla de asesorar y apoyar a los centros educativos.

Las reformas Neoliberales de los noventa permitieron avanzar en la descentralización del sistema educativo. Los supervisores se convirtieron en figura clave para la organización lógica y la articulación de la macro y la micro políticas institucionales. La Ley Federal de Educación N²4195 (1993), modifica la estructura académica y normativa.

Las grandes reformas estructurales del sistema educativo en Argentina en los últimos veinte años, posibilitaron cambios muy significativos tanto en la organización como en el perfil de los inspectores zonales. El número de inspectores de cada nivel fue uno de los más importantes cambios en casi todas las provincias.

Glickman (1992), sostiene que existen debates entre las concepciones de las funciones del inspector escolar, pero en este período se acentúan las tendencias que ponen en consideración privilegiar los modos del hacer del supervisor desde la función de asesoramiento, colaboración y orientación a todos los actores sociales de las instituciones educativas a fin de lograr la mejora esperada.

En 1994, en el encuentro de Supervisores Docentes en Argentina, se reclamaba mayor participación en la toma de decisiones y la necesidad de poner como prioridad lo pedagógico. A pesar de los acuerdos y las nuevas regulaciones, coexisten varios modelos de supervisión que tienen que ver con el controlar y vigilar en contrapartida con el asesorar y orientar pedagógicamente.

Los años 90 trajeron consigo distintos cambios educativos que evidenciaron otra tensión en cuanto a los modelos de supervisión, la misma ya se venía manifestando internacionalmente. Se trata de modelos más generalistas, con miradas integrales sobre la escuela y modelos de supervisión focalizada en el trabajo en el aula, también aparecen los modelos de supervisores que se especializan en distintas áreas curriculares o modelos mixtos formados por supervisores generalistas y especialistas. Es este último el caso de Argentina.

Epistemológicamente se atraviesa un período donde se buscan por diferentes caminos fundamentos a la realidad. Así Popper con su crítica al positivismo, Bachetard desde el racionalismo crítico, Kulm con la introducción de la historia de las ciencias, Gödel y sus sistemas complejos, entre otros que entran en crisis:

"Brecha en lo Real, abriendo una hiancia inaccesible a la inteligibilidad; brecha en la lógica, que deja de ser apta para cerrarse demostrativamente sobre sí misma; por estas dos brechas, se efectúa una hemorragia de aquello que sigue llamándose lo Real, y la pérdida irreparable de los fundamentos de aquello que hay que continuar llamando Conocimiento." (Morin,1988, p.24).

Dussel (1995) afirma que se van configurando diferencias provinciales en cuanto a la relación de la dimensión política del supervisor con la dimensión técnica. Generalizando, la mayoría de las provincias adhiere a la visión de la constitución de la dimensión política como un componente fundamental de la identidad del supervisor, que se asocia especialmente a las acciones supervisivas de la provincia, que es donde la dimensión política gana mayor intensidad, porque, es allí donde se hace más visible la intermediación entre lo prescripto por las políticas educativas y la planificación o el plan que el supervisor proyecta para todas las instituciones educativas a su cargo.

En la tercera etapa, o etapa del facilitador/auditor, el inspector debiera gestionar para evitar la exclusión y promover el sostenimiento de las trayectorias escolares, habilitando las preguntas para la reflexión sobre las prácticas de enseñanza (Veleda, 2011).

El inspector tiene que lograr una intervención situada, debe conocer las escuelas, sus prácticas, la calidad de los aprendizajes y las condiciones de enseñanza que la escuela habilita, no desconocer las debilidades, pero ver a cada institución desde sus posibilidades y acompañarla en la mejora. 
Desde el paradigma de la complejidad, la posibilidad de generar las condiciones para que desde la Supervisión se desarrollen prácticas de ciudadanía democrática que permitan desarrollar prácticas de conocimiento reales en cada aula de cada Zona Escolar de tal modo que todos los estudiantes alcancen aprendizajes de calidad, aprendizajes que les permitan mejorar la calidad de sus vidas tanto en lo personal como en lo social. Morin hace referencia al paradigma de la complejidad señalando:

...un paradigma de distinción/conjunción que permita distinguir sin desarticular, asociar sin identificar o reducir. Este paradigma comportaría un principio dialógico y translógico, que integraría la lógica clásica teniendo en cuenta sus límites de facto (problemas de contradicciones) y de jure (límites del formalismo). (Morin, 1994, p.34).

Ante las diversas modalidades se mantuvo un rasgo común en la mayoría de los países como representante de los estados nacionales en el control de los sistemas educativo, en general (Glanz, 1997). Distintos autores (Carron y De Grauwe, 2003) analizaron los cambios en los modelos de organización de la supervisión y encuentran fuertes debates por parte de los países sobre los modelos a adoptar, especialmente con los siguientes aspectos:

Las estructuras de supervisión: Varios optan por estructuras centralizadas, o sea que dependen del poder central como en el caso de Francia, pero, por otro lado, en otros países se favorecen estructuras descentralizadas, los supervisores dependen de los organismos locales; son elegidos por la comunidad educativa o por instancias colegiadas al interior de los distritos o regiones como en el caso de Inglaterra. $Y$ la organización de la supervisión: independientemente de si son niveles centrales o locales, todos los países optan por marcar como formas de organización de la supervisión. Pudiendo ser por niveles educativos, por áreas del conocimiento, por sector público o privado o por combinaciones que incluyen las distintas alternativas. Existen países que organizan la supervisión sobre la base de supervisores generalistas que tienen una mirada sobre la institución como totalidad o supervisores especialistas que se focalizan en una materia o área de conocimiento.

En su mayoría los países estructuran la supervisión por niveles educativos ya sea desde lo público como desde lo privado. Otra opción de preferencia es la supervisión generalista escoltada por asesores técnicos en las materias o áreas de conocimiento específicas según el nivel educativo (Estados Unidos, Reino Unido, Brasil, España).

La tensión entre la dimensión política y la dimensión técnica de la supervisión: La dimensión política del rol del supervisor hace referencia a la mediación e intermediación entre los niveles centrales de conducción política, las regiones y distritos, las escuelas y sus contextos. Dando al supervisor un rol de "mediador", "puente" o "bisagra" que brinda un importante componente político en su función, actuando como promotor u obstaculizador de los lineamientos de las políticas educativas. El supervisor es, al mismo tiempo, un especialista y un experto en la problemática educativa en general y en la del nivel a su cargo en particular, lo que lo constituye en un cuadro técnico con significativa incidencia en la implementación de los proyectos educativos.

La función predominante en el trabajo del supervisor: Cada país opta por privilegiar la función de evaluación y control o la función de asesoramiento y orientación a las escuelas. La gran mayoría de los países opta por una combinación de ambos tipos.

Las estrategias de selección de los supervisores: Algunos países los seleccionan mediante concursos internos realizados entre los directivos a cargo de las escuelas (Argentina) mientras que otros realizan selecciones abiertas entre profesionales de la educación sin que sea imprescindible haber sido o estar desempeñándose como directivo (España, Noruega).

También, el estudio llevado a cabo por la DiNIECE (2013), nos cuenta que, en Gran Bretaña, las visitas de los supervisores son negativas por el estrés que provocan en directivos y docentes. En España se sostiene la necesidad de vínculo de horizontalidad y cooperación entre supervisores y los demás actores institucionales. En Brasil el trabajo de supervisión es complejo y de gran heterogeneidad por las reformas educativas. En Chile se privilegia la dimensión estrictamente técnica de la supervisión, siendo un órgano colegiado y en un nivel central. 
En México, existen numerosas dependencias estatales y federales que no logran coordinar entre sí y que imprimen un alto grado de complejidad en la función del supervisor.

Así, generalizando, en todos los países la supervisión escolar está sumergida en un proceso de transición que va desde la función individual y de control sobre la escuela a la supervisión como proceso de construcción conjunta y colegiada.

En Córdoba se proclama una convocatoria a Concurso de Títulos, Antecedentes y Oposición en cargos de inspectores técnicos de educación inicial y educación primaria en el año 2000. En esa convocatoria se redefinen las características del perfil del supervisor, que se resumen en la posibilidad de promover procesos de cambio a través de la contextualización de las políticas públicas provinciales y nacionales; favorecer la autonomía de las instituciones; ser capaz de evaluar a las escuelas desde todas sus dimensiones y con el accionar de todos los actores institucionales y generar proyectos de gestión supervisiva para organizar el desarrollo educativo regional.

Este nuevo perfil resignifica el trabajo de control del inspector y muestra un enfoque más democrático orientado a promocionar la autonomía de las escuelas en el marco del mejoramiento de la calidad educativa a través de la gestión institucional. Consecuentemente, el proceso de dicho concurso da indicios de la construcción de otro modelo de supervisión que instala una cultura de trabajo que sostiene la atención a la especificidad de las problemáticas más acuciantes; fortalecimiento de los canales de comunicación; concentración de esfuerzos para promover una educación de mayor calidad por la vía del consenso, el diálogo, la concertación y la participación.

En el año 2011, la Secretaría de Educación de Córdoba, a partir de la lectura que realiza de las problemáticas sobre la supervisión escolar, elabora un documento para que sea trabajado por los supervisores. El documento titulado "Una invitación a pensar el trabajo del Supervisor" se propone construir distintos sentidos en la gestión de los supervisores para resignificar su tarea hacia las definiciones que la política educativa.

Estos son, algunas líneas de la historia y de otros lugares y formatos que nos permiten abarcar un poco más, esta idea de quién es el inspector de zona o el supervisor escolar.

\section{Los sentidos desde lo prescripto}

Continuando esta construcción de sentido de la identidad de inspector de zona escolar, se avanza a fin de definir en la actualidad, en Argentina y en Córdoba, qué figura se describe desde la legislación vigente.

La Ley de Educación Nacional², sancionada en el año 2006, vigente actualmente, establece en su Artículo 12 que "El Estado Nacional, las Provincias y la Ciudad Autónoma de Buenos Aires, de manera concertada y concurrente, son los responsables de la planificación, organización, supervisión y financiación del Sistema Educativo Nacional".

Esta Ley establece también la organización de la supervisión, principalmente hace referencia a la organización de sus funciones, a la conformación de las estructuras de supervisión en los distintos niveles y a la identidad profesional definida por los conocimientos teóricos y metodológicos que forman el perfil deseado para el desempeño del supervisor.

El Artículo 73 de dicha ley plantea la formación docente como objetivo de política nacional. Se trata de jerarquizar y revalorizar la formación de docentes para mejorar la calidad de la educación. Para ello el Gobierno Nacional crea una capacitación semipresencial, en el marco del Plan Estratégico Nacional 2016-20213.

La Resolución CFE №338 (2018) sostiene que el rol de los inspectores es fundamental para lograr las mejoras esperadas en todas las escuelas de su zona, promoviendo la calidad de la enseñanza y los aprendizajes, y garantizando la trayectoria educativa de los alumnos. Los supervisores son quienes pueden facilitar la

2. Ley $N^{\circ} 26.206$, Ley de Educación Nacional, Argentina, sancionada el 14 de diciembre de 2006 y promulgada el 27 de diciembre de 2006. Extraída de http://www. secgral.unsl.edu.ar/wp-content/uploads/docs/Ley-26206-de-Educacion-Nacional.pdf

3. Anexo Resolución CFE №285 de 2016. Plan Estratégico Nacional 2016-2021. Extraída de http://www.bnm.me.gov.ar/giga1/documentos/EL005360.pdf 
construcción de consensos entre escuelas, generar instancias de cooperación horizontal y promover la articulación entre niveles educativos. La misma, presenta un documento que tiene por objetivo describir los lineamientos federales para el desarrollo profesional de los equipos directivos y de supervisores/inspectores fruto de un acuerdo federal construido en una comisión técnica con referentes de gestión educativa elegidos por cada jurisdicción, donde se declama como principios pedagógicos a: 1. Desarrollo de capacidades y actitudes. 2-Foco en la práctica. 3-Comunidad de práctica. 4-Práctica reflexiva. 5- Evaluar para aprender.

Hace referencia también a cuatro ejes temáticos a tener en cuenta para la formación de directivos e inspectores: Eje 1: Desarrollo de la organización escolar. Eje2: Acompañamiento del proceso de enseñanza, aprendizaje y evaluación. Eje 3: Liderazgo del desarrollo profesional, Eje 4: Construcción de la comunicación educativa y el vínculo con el contexto.

Por su parte, la Ley de Educación de la Provincia de Córdoba, N9870 (2010)4 , en su artículo 104 declama que los supervisores integran un equipo técnico docente de las distintas direcciones del sistema y desempeñan la tarea de asesorar y apoyar a las instituciones educativas para el mejor desarrollo de su proyecto institucional. También define el acceso al cargo a través de estatutos y normas que regulen la carrera.

En este sentido, en el año 2015 Córdoba sanciona la Ley $N^{\circ} 10.237$ de Concursos ${ }^{5}$ con el afán de regular los Concursos de ascenso a cargos jerárquicos, la misma modifica al Decreto Ley №1910 (1957) y su Decreto Reglamentario es el 930 (2015). Los cambios más sustanciales tienen que ver con la garantía de la continuidad de las convocatorias ya que crea la Comisión permanente de concursos.

A partir de la Resolución N¹020 (2016) de la Dirección General de Educación Primaria del Ministerio de Educación de la Provincia de Córdoba, correspondiente al 2 de noviembre del 2016, se determina el perfil del Supervisor de hoy:

El desafío de ser supervisor hoy requiere una sólida formación profesional para el ejercicio de una gestión pedagógica que tenga como prioridad a los estudiantes, garantizando la inclusión y calidad educativas a las que tiene derecho todo ciudadano, en cualquier etapa de su vida, y haciendo confluir el trabajo en equipo de cuerpo directivo, los docentes, las familias y otros actores de la comunidad. Del mismo modo, el supervisor es garante de una gestión administrativa que asegure el efectivo cumplimiento de los principios y objetivos establecidos en la legislación educativa, sin perder de vista el vínculo con la cultura, la política y la sociedad contemporáneas. (p.7).

Poner en marcha este desafío implica proyectarnos como zona escolar desde la mirada complementaria de los distintos actores que la componen, rescatar el proceso experimentado por cada uno, poniéndolo como fuente de conocimiento común y autoridad confiable.

Es necesario que el Inspector de zona escolar se perfile según lo determinado por la Resolución antes mencionada logrando en cada dimensión formativa una intervención que asegure los lineamientos trazados por las políticas Educativas del Estado Provincial, adecuándolas a las necesidades de la zona, al desarrollo de las capacidades de los directivos y de las escuelas para el logro de la calidad en los aprendizajes de todos los estudiantes.

\section{Para ello deberá considerarse:}

\section{Desde la Dimensión Cultural:}

El Supervisor necesita de una formación cultural amplia que potencie una mirada integral y contextualizada, a través de la cual sea capaz de ofrecer aportes para comprender la impronta singular de las transformaciones culturales contemporáneas en todas y cada una de las instituciones a su cargo. Este conocimiento le posibilitará orientar cambios institucionales que fortalezcan a las instituciones educativas como uno de los lugares imprescindibles para la formación de la ciudadanía en un mundo cambiante e incierto, contribuyendo

4. Ley N`9870 de Educación de la Provincia de Córdoba, sancionada en diciembre de 2010. Extraída de https://filadd.com/doc/ley-9-870-de-educacion-de-la-provincia-de-cordoba

5. Ley ํㅜ 10.237 de Concursos para la cobertura en condición de titulares de cargos directivos y de inspección en el ámbito del Ministerio de Educación. 2015. Extraí da de https://www.cba.gov.ar/wp-content/4p96humuzp/2016/03/LEY-10237-2014.pdf 
a formar sujetos responsables y participativos, al mismo tiempo que críticos y creativos. (Resolución N 1020 DGEP ANEXO II, 2016 p.8).

\section{Desde la dimensión política:}

La dimensión política de la función del supervisor demanda de él una formación que lo involucre activamente tanto en la comprensión / interpretación de las políticas educativas, como en el análisis de las políticas institucionales de las escuelas a su cargo, para proyectar acciones de contextualización, mediación y adaptación de las macro políticas, según las posibilidades del contexto social de las instituciones. Dentro de la dimensión política del Estado y las instituciones educativas, hay que reconocer el componente administrativo que conlleva siempre la implementación de las políticas y el sostenimiento del conjunto del Sistema Educativo. Toda política pública y el sistema educativo necesitan de un conjunto de organismos que componen la burocracia estatal y que tiene como función asegurar los mecanismos legales y legítimos para el funcionamiento diario del sistema y de las escuelas. Leyes, decretos, reglamentos, estatutos son los componentes burocráticos que permiten el gobierno diario de las instituciones. Representan los reaseguros legales para consolidar formas de funcionamiento y dirimir algunos conflictos. Representan, por un lado, límites a las conductas individuales y al mismo tiempo, aseguran protección ante irregularidades o excesos que puedan cercenar los derechos individuales y colectivos. El componente administrativo del sistema y de los centros educativos requiere del Supervisor un conocimiento actualizado de los requisitos legales para saber utilizarlos ante situaciones diarias y extraordinarias. Este conocimiento no necesita ser exhaustivo, sino lo suficientemente global y significativo para saber cuál recurso se debe utilizar o dónde encontrar la información correspondiente. Además, el supervisor debe contar con suficiente amplitud de criterio para plantear con pertinencia los problemas más delicados ante organismos y especialistas que colaboran y aconsejan en la labor diaria de los agentes del sistema, cuya tarea supone mayor responsabilidad. (Resolución N¹020 DGEP ANEXO II 2016 p.9-10).

\section{Desde la Dimensión Pedagógica:}

Es la orientación pedagógica la que debe marcar el rumbo en la configuración de las formas de hacer escuela. La pedagogía como teoría y práctica de la educación es prescriptiva respecto de las relaciones que se desarrollan en los centros educativos. Crea orden, relaciones y autoridad en un registro ético. Por orientación pedagógica se entiende, entonces, a las formas de resolver las cuestiones que se presentan en las relaciones al interior de las instituciones cuyas finalidades son educativas, teniendo siempre como norte la formación de estudiantes críticos, responsables, creativos y fundamentalmente éticos. Dicho en otras palabras, lo que distingue a la institución escolar de otras instituciones sociales es la función de transmisión y adquisición de conocimientos para formar sujetos que otorguen valor a los aprendizajes y a las prácticas de convivencia democrática. A pesar de la crisis de la escuela actual, ninguna otra institución de la sociedad está en condiciones de intentar estos efectos de manera sistemática y prolongada. El eje vertebrador que enmarca esta función es el currículo. El currículo es el soporte identitario de la escuela como institución social, entendido, en un sentido amplio como lo define Alicia de Alba, como una "síntesis de elementos culturales (conocimientos, valores, costumbres, hábitos) que conforman una propuesta política-educativa”. La interacción entre sujetos, institución, currículo, trayectoria y contexto, brinda un acercamiento a la trama institucional donde los límites y las articulaciones de cada uno de los componentes, tanto estructurales como vinculares, son de difícil aprehensión si no media una reflexión analítica que permita comprender los distintos procesos que, en el seno de las instituciones, se construyen. Lo anterior alude a la necesidad de reflexión colectiva sobre el sentido que para cada escuela y sus integrantes revisten los componentes señalados. El trabajo en equipo y la construcción de un proyecto escolar de mejora que vertebre a todos ellos, dan cuenta de la orientación pedagógica de la institución. En este sentido, la autoevaluación institucional es fundamental al producir conocimiento que permite abordar las fortalezas, oportunidades, dificultades y los conflictos, habilitando mejores formas de trabajar e interactuar. Otra cuestión que posibilita las mejoras institucionales es el trabajo en red con otras instituciones escolares y de la comunidad, en la medida que la comunicación de experiencias y decisiones pueden producir una sinergia que movilice intereses y prácticas. (Resolución N¹020 DGEP ANEXO II, 2016 p.11).

Este es el panorama de la identidad del supervisor a través de las leyes vigentes en la jurisdicción donde se inserta esta investigación, 


\section{Roles y funciones del Inspector}

Como ya queda especificado en los ítems anteriores, las funciones del inspector de zona escolar han ido cambiando con el paso del tiempo y según las políticas de gobierno por las que eran atravesadas. En esta oportunidad, la finalidad está puesta en ampliar la mirada internacional sobre roles y funciones de la supervisión.

En cuanto a las funciones del supervisor, García Martínez (2011), hace hincapié en una mirada constructivista que debe formar el inspector escolar. Cita a Glickman (1990) para definir a la supervisión, desde lo académico, como la función escolar que busca la mejora de la educación a través de la asistencia directa a los docentes, como así también, desde el desarrollo del currículum y desde el avance personal y la consolidación de los grupos. Este autor además, conceptualiza la función de vigilancia, control y cuidado del status quo de la escuela, que es el modelo que aún sigue vigente en México.

Por su parte, Mogollón de González (2006) nos habla de la función supervisora con técnicas administrativas, sociales, de asistencia y mediación. Esta investigación tiene como propósito presentar la aproximación a un modelo para las funciones de la supervisión escolar en Venezuela. Las categoriza de la siguiente manera:

- Funciones Técnicas, las cuales abarcan el currículo, la actualización docente, los planes y programas, los recursos de aprendizaje y los procesos de organización administrativa.

- Funciones Administrativas: se trata de la tarea de habilitar los procesos de asesoramiento, planificación, supervisión y evaluación donde también se incluyen los procesos administrativos.

- Funciones Sociales: son las que tienen hacen referencia a los ámbitos de comunidad educativa y comunicación.

- Funciones de asistencia: abarcan el asesoramiento pedagógico, las relaciones humanas e instituciones.

- Funciones de mediación: abarcan los ámbitos donde se despliega la normativa y reglamentación jurídica.

Pero es Camacho Prat (2014), de España, quien muestra una mirada más integral y más acorde a los datos investigados, ella se sintetiza en:

La supervisión como comprobación y control documental con el director. La evaluación como participación en la misma, siempre sometida a ideologías del poder político. El asesoramiento limitado a la mera recomendación jurídica a los directivos. La emisión de informes, importantes para la mejora muchas veces solicitados por la administración. La mediación y el arbitraje, junto al exceso de tareas burocráticas y rutinas administrativas.

Esta mirada se completa con la perspectiva de acompañar que imprime Erazo Juarez (2013). Para él, las funciones del supervisor se definen en las siguientes:

La función de asesoría y acompañamiento docente, se resumen en la gestión, la realización de capacitaciones de actualización de los conocimientos y el ofrecimiento de los conocimientos que tiene el inspector para el desarrollo profesional de los docentes.

La función de evaluación en forma de retroalimentación sobre los desempeños para reorientar el proceso educativo. El carácter que imprime la evaluación es integral, relacional y complementaria, es pensar la realidad de los centros educativos, analizarlos y convertirlos en problemas a resolver.

La función de mediación y resolución de problemas facilitan el diálogo y la negociación, con un liderazgo democrático cumpliendo roles de mediador, orientador, investigador y promotor social. 
Estos autores seleccionados dan cuenta del resto de la literatura académica que circula, por lo que se consideran suficientes para evitar repeticiones o superposiciones.

\section{Las prácticas del inspector}

EI CIPPEC (2017), a través de un mapa virtual, presenta prácticas potentes de supervisión en una experiencia interactiva cuyos principales referentes son Rivas y Furman.

Un ejemplo de la recopilación del "Mapa de exploración de la innovación educativa” que realizan es el siguiente:

Nivel Primario Portafolio: Memoria pedagógica zonal Gestión institucional "La necesidad de construir memoria pedagógica zonal para que no se pierdan las experiencias valiosas y los momentos únicos vividos en cada rincón de las escuelas, se decide armar un portafolio con narrativas de directivos y docentes. La consigna simplemente es hacer cortes evaluativos institucionales, de carácter trimestral, y rescatar ese momento mágico que hizo la diferencia a través de narrativas. Lo resultante se trabaja en diferentes reuniones de personal donde se realiza el análisis reflexivo de las prácticas de gestión y áulicas a través de ellas. En otras oportunidades también sirven como lecturas motivadoras para introducir ciertas temáticas a debate zonal. Como extensión a otras instituciones, se ha realizado una copia de las recopiladas durante el primer año de implementación y se la entregó al Instituto de Formación Docente de la localidad como material de trabajo." CIPPEC (2019).

También avanzan el tema desde las prácticas del supervisor, Hirschberg (2013), determina tres dimensiones desde dónde se realizan las tareas del inspector escolar: dimensión política, como transmisor e implementador de la política; territorial, en su interacción con los actores de su jurisdicción, autoridades municipales, organizaciones de salud, minoridad y acción social, no gubernamentales, entre otras y la dimensión pedagógica como responsable de la implementación de las estrategias de enseñanza y aprendizaje desde las innovaciones pedagógicas.

Erazo Juarez (2013), de Honduras, presenta desde los manuales del SINASEH los tipos de supervisión como prácticas que pueden identificarse con la mirada de la supervisión correctiva, la que se focaliza en buscar y encontrar los errores y debilidades y así diagramar estrategias colectivas de solución. Por otro lado, presenta la supervisión constructiva que brinda soluciones en forma integral, esta incluye al proceso de enseñanza y de aprendizaje, hace hincapié en el desarrollo de las capacidades de los docentes para mejorar la tarea. También hace referencia a la supervisión creativa, que es aquella que busca la estimulación de los docentes para encontrar soluciones a los problemas que se van presentando mediante la superación constante. Y, por último, presenta la supervisión preventiva que intenta evitar los problemas antes que aparezcan, buscando fortalecer la formación de los docentes.

Y, por último, se cita del mismo autor, lo que él denomina técnicas de supervisión. Con técnicas de supervisor refiere a procedimientos ideados para recoger datos en las prácticas de los diferentes autores: reuniones, entrevistas privadas, observación de clases, visitas, trabajos en equipos, grupos focales, entre otras. Y otras técnicas que son denominadas indirectas y tienen por finalidad brindar datos sobre la situación que se pone en estudio: currículum del docente, planes de clases, aprendizaje de los estudiantes, posibilidades de integración de la enseñanza, reconocimiento de las características de los estudiantes, cuaderno de temas, horarios, materiales y recursos, portafolio, y muchas otras.

El español, Camacho Prat (2014), nos presenta las prácticas profesionales del supervisor. Coloca a la visita a la escuela como la más relevante entre ellas, considera que el contacto con la realidad es la base de su trabajo. Otras tareas que menciona son la revisión del plan de actuación y las instancias de parte y oficio. La tarea administrativa como las incidentales ocupan mucho tiempo de la agenda.

Delgado (2019), que analiza la situación de la supervisión en la provincia de Chaco, Argentina, expresa que como organización continúa predominando un modelo de gestión normativo y coexisten elementos del modelo estratégico participativo. 
Casal (2019) habilita una mirada inclusiva a las prácticas del supervisor, pone en centro las trayectorias escolares. Transmite que, en los tiempos actuales, el trabajo conjunto en clave de sistema, sostiene y potentizan las prácticas y propone imaginación conjunta y responsable.

Este recorrido por los aportes hacia las prácticas que lleva a cabo el inspector de zona escolar, deja claro la complejidad de las mismas.

\section{Otros aportes que complementan conceptos}

Existen otros estudios que nos permiten seguir pensando la identidad del inspector de zona escolar desde perspectivas diferentes.

Pozner (2007) hace referencia a la profesionalidad del supervisor:

La nueva profesionalidad en la supervisión de la educación, incluye el desarrollo de competencias como la reflexión, creatividad, capacidad de decisión y acción, abstracción, pensamiento sistémico, experimentación y capacidad de trabajar en equipo; a su vez estas deben ser capaces de transferirse y aplicarse a todas las situaciones, problemas y procesos de acción, es decir deben vincularse con su contexto e incluso con el desempeño colectivo de una profesión. Y convendrá considerar los puntos críticos que aparecen en el tránsito de modelos centralizados a otros más flexibles. Su identificación y análisis obliga a pensar la necesaria reconfiguración de los sistemas educativos, sin la cual las iniciativas de cambio pueden fracasar o paralizarse. (p.18).

Se adhiere al posicionamiento de Soler Fiérrez (1994), quien afirma que la supervisión cuenta con suficiente consistencia interna y legitimidad para poder considerarla como una especialidad de las ciencias pedagógicas. Presenta el término de metasupervisión, haciendo referencia al sentido, procedimiento y efecto de su propia práctica. Y con la mirada de Aguerrondo (2012) y Aguilar Santos (2005), quienes lo consideran un actor clave con oportunidades de innovación y transformación del sistema.

El inspector de zona, es entonces, un actor estratégico que trabaja el desarrollo de las relaciones entre las instituciones de la zona y la decisión política de la gestión convirtiéndose así en un facilitador en la toma de decisiones. Es el inspector de zona escolar el que en muchos casos propone líneas de trabajo según lo que observa en territorio, que pueden contribuir con el funcionamiento y mejoramiento de las instituciones, ya que es quien mejor conoce las escuelas y su población, quien debe hacer efectivas las decisiones de la gestión política y quien debe comunicar a las autoridades del sistema educativo las situaciones de los establecimientos, los problemas que enfrentan y las acciones que en ellos se llevan a cabo.

Torres Hernández (2007), de México, es el que inicia la presentación de una gran necesidad de mostrar la función del supervisor desde la complejidad que la misma representa. Lo hace desde el desarrollo de la mirada a los procesos y actividades diferentes que desarrolla, descubriendo los diferentes roles que juega y las implicancias que su presencia provoca tanto en los procesos como en cada uno de los sujetos partes del sistema. Así mismo, hace referencia a la construcción y reconstrucción colectiva de todos los saberes sobre la inspección escolar, los supuestos, los prejuicios y las teorías desde los cuales son sustentados.

Amplía también el concepto desde la perspectiva de pensar la supervisión como un espacio de gestión compartida entre los actores de la zona, convirtiéndose así en parte de la corresponsabilidad en la discusión curricular a partir de la contextualización que emerge de las necesidades locales. Se reconoce así a la inspección como un elemento organizativo que pertenece a un sistema educativo, con las funciones particulares que cobra mayor sentido en la complejidad de interacciones y relaciones que se establecen con cada uno de los miembros de la zona escolar: directivos, docentes, padres, estudiantes. Es así que se puede pensar en la inspección como una institución que representa los principios de organización y de funcionamiento de las escuelas como orden de control que permite mantener un orden socioeducativo. 


\section{Conclusiones}

Y en este entramado de voces que permiten entender la identidad del supervisor desde su esencia, desde su historia, desde sus funciones y sus prácticas, se sigue intentando responder a ¿cómo son los modos diferentes en que se desarrolla la función formadora del inspector de zona escolar?

Ser supervisor hoy permite pensar y accionar en miras de un horizonte de constante mejoramiento. Es por ello que todo profesional docente debe creer y buscar comprometidamente el cambio, así como la formación para llevarlo a cabo y de la constancia para lograrlo, descubrir en el quehacer diario, la pasión por lo que se hace. Saber decidir, realizar, reinterpretar, generar diálogos, traducir, viabilizar, tener equilibrio reflexivo, enhebrar, sedimentar, evaluar y balancear.

Sus funciones de acompañamiento y asesoramiento a los equipos directivos de su zona -dimensión pedagógica del rol directivo- habilitan la articulación entre las políticas educativas y la realidad de las escuelas. Esto le permite gestionar información tanto de los estamentos del sistema como la recogida de datos en terreno. Es un lugar estratégico donde se enlazan la construcción de las políticas educativas y su desarrollo, permitiendo su contribución con la macropolítica haciendo posible el mejoramiento en las micropolíticas. Así, los inspectores se convierten en agentes de justicia educativa desde un lugar que no es neutral, actúan desde la redistribución y el reconocimiento en el entramado de la desigualdad social vigente.

En esta práctica pedagógica, el sostenimiento de las trayectorias escolares de todos y cada uno de los estudiantes de la zona es uno de los desafíos prioritarios desde dónde se piensa la intervención del inspector.

Consecuentemente, es un constructor de ámbitos democráticos, de conquistas de derechos en contextos de ruptura de los lazos sociales y extensión de los conflictos interpersonales. Para ello podrá formar buenos liderazgos de directivos que actúen en cada escuela, usar protocolos sobre qué hacer ante diversos conflictos y tener respuestas para generar la cohesión social preventiva y no actuar solo cuando aparecen los conflictos.

Entonces, siguiendo a Nicastro (2019), se define a la inspección de zona como la práctica especializada y situada, haciendo referencia a puntos importantes a considerar: por un lado, el inspector atiende constantemente situaciones particulares en el marco de regulaciones generales, siendo ese el foco de su función. Es el modo de llevar adelante la tarea, la que se concreta produciendo situaciones de colectivización. También es necesario pensar que el trabajo del inspector no es solo estar alerta, diagnosticar o evaluar las condiciones institucionales existente, también es producir constantemente esas condiciones institucionales para que sea efectivo el desarrollo de esas prescripciones. Y son esas condiciones son las que determinan los diferentes modos que asume la función formadora del inspector.

\section{Referencias}

Aguilar Santos, E. (2005). La Inspección en las distintas concepciones y sistemas pedagógicos: características y funciones. (Tesis de Maestría). Universidad Pedagógica Nacional Francisco Morazán. Trujillo, Honduras. Recuperada de file:///D:/Downloads/opciones-y-condicionantes-de-la-supervision-educativa-en-eldepartamento-de-olancho.pdf

Camacho Prats, A. (2014). Funciones y quehaceres de los inspectores de Educación en Baleares. Un estudio de casos. (Tesis doctoral). Universidad de Barcelona, Barcelona, España. Recuperada de https://dialnet. unirioja.es/servlet/tesis?codigo $=45435$

Carron, G. y De Grauwe, A. (2003). Cuestiones de actualidad en supervisión: una revisión de la literatura. París, UNESCO- International Institute for Educational Planning. Castro de Pérez, Abigail; Mesa, Darlyn; Guzmán, José Luis y Picardo Joao, Oscar (1999). La reforma de la historia y la historia de la reforma. (La Reforma Educativa en marcha de El Salvador). Informe de investigación. San Salvador.

Casal, V. (2019). Propiciando prácticas inclusivas desde la Supervisión Escolar. Academia. Recuperado de https://uba.academia.edu/VanesaCasal 
Delgado, P. (2019). Modelos de gestión educativa en las prácticas de supervisores de EGB chaqueñas. (Tesis de Licenciatura) Universidad Nacional del Nordeste. Resistencia, Chaco, Argentina. Recuperada de https:// www.academia.edu/26854794/Modelos_de_gesti\%C3\%B3n_educativa_en_las_pr\%C3\%A1cticas_de_ supervisores_de_EGB_chaque\%C3\%B1as

Decreto Ley 1910. Estatuto del docente primario y preprimario, Córdoba, Argentina (1957).Extraído de: http:// portaldetramites.cba.gov.ar/doc/MINED/cordoba_decretoley1910.pdf

DiNIECE, Ministerio de Educación, Presidencia de la Nación (2013). "La supervisión educativa en nuestro país: El trabajo del supervisor en cinco jurisdicciones". Serie la Educación en Debate. Recuperada de: http:// www.bnm.me.gov.ar/giga1/documentos/EL006494.pdf

Dirección de Investigación e Innovaciones Educativas (1994) Seminario-Taller "La Gestión del Supervisor" Ministerio de Educación y Cultura. Gobierno de Córdoba.

Dussel, I. (1995). Pedagogía y Burocracia. Notas sobre la historia de los inspectores. Revista Argentina de Educación, №. 23. Asociación de Graduados en Ciencias de la Educación, Buenos Aires. Recuperado de http://www.die.cinvestav.mx/Portals/die/SiteDocs/Investigadores/IDussel/ArticulosDussel/ PedagogiayBurocracia-.pdf?ver=2013-09-30-161517-873

Ediciones Bellaterra.

Erazo Juárez, O.A. (2013). Incidencia de la Supervisión Educativa y Acompañamiento Pedagógico de los Docentes que laboran en la Escuela Normal Mixta "Matilde Córdova de Suazo" de la Ciudad de Trujillo, departamento Colón. (Tesis de Maestría). Universidad Pedagógica Nacional Francisco Morazán. Trujillo, Honduras. Recuperada de http://www.cervantesvirtual.com/nd/ark:/59851/bmc612w8

García Martínez, M. (2011). Funciones pedagógicas de la Supervisión escolar en educación primaria del distrito federal. (Tesis de licenciatura). Universidad Pedagógica Nacional, México. Recuperada de https:// es.scribd.com/document/148979195/Supervision-Escolar

Glanz, J. (1997). El Tao de la supervisión: ideas taoístas sobre la teoría y la práctica de la supervisión educativa. Revista de currículo y supervisión, Vol. $12 \mathrm{~N}^{\circ} 3,193$ - 211. Recuperado de https://search. proquest.com/openview/41919d2eef63f8c2dd250dfe9f771033/1?pq-origsite=gscholar\&cbl=35996

Glickman, C.D. (1992). Supervision in tranisition. Alexandria, VA: ASCD

Gvirtz, S. y Podestá, M. E. (Ed.). (2012). El rol del supervisor en la mejora escolar. Buenos Aires: Aique Educación.

Ley de Subvenciones Nacionales N 463 (1871) Extraída de https://www.preceden.com/timelines/373649-leyde-educacion-nacional-argentino--resumen-

Ley de Educación Común de la provincia de Buenos Aires (1875) Extraída de http://www.memoria.fahce.unlp. edu.ar/art revistas/pr.1051/pr.1051.pdf

Ley 1420 de Educación Común y su Reglamento del Consejo Nacional de Educación, Argentina, (8 de julio de 1884). Extraída de http://www.bnm.me.gov.ar/giga1/documentos/EL002646.pdf

Ley 14.473. Estatuto del docente. (12 de septiembre de 1958) publicada en el Boletín Oficial el 27 de septiembre de 1958. Extraída de http://www.bnm.me.gov.ar/giga1/normas/8178.pdf

Ley 1426, Ley Orgánica de Educación Primaria, Córdoba, Argentina (1896). Extraída de file:///C:/Users/rasan/ Downloads/12216-32303-1-PB\%20(1).pdf

Ley 8114, Ley General de Educación de la Provincia de Córdoba (1991). Extraída de http://www.bnm.me.gov.ar/ redes federales/snie/pais/cordoba/normativa/docs/cordoba.pdf

Ley 24.195, Ley Federal de Educación de la República Argentina, (14 de abril 1993) promulgada el 29 de abril de 1993. Extraída de https://www.buenosaires.gob.ar/sites/gcaba/files/ley 24195.pdf 
Ley 26.206, Ley de Educación Nacional, Argentina (14 de diciembre de 2006) promulgada el 27 de diciembre de 2006. Extraída de http://www.secgral.unsl.edu.ar/wp-content/uploads/docs/Ley-26206-deEducacion-Nacional.pdf

Anexo Resolución CFE №285 (2016). Plan Estratégico Nacional 2016-2021. Extraída de http://www.bnm. me.gov.ar/giga1/documentos/EL005360.pdf

Resolución CFE N³38 del 2018 Extraída de http://www.bnm.me.gov.ar/giga1/normas/RCFE 338-18.pdf

Ley N9870 de Educación de la Provincia de Córdoba, (2010). Extraída de https://filadd.com/doc/ley-9-870de-educacion-de-la-provincia-de-cordoba

Ley $N^{\circ} 10.237$ de Concursos para la cobertura en condición de titulares de cargos directivos y de inspección en el ámbito del Ministerio de Educación (2015). Extraída de https://www.cba.gov.ar/wpcontent/4p96humuzp/2016/03/LEY-10237-2014.pdf

Decreto Ley 1910. Estatuto del Docente Primario y Preprimario. (1957). Leyes de Educación, Provincia de Córdoba. (Recopilado por Carbó, E.)

Resolución N¹020 de la Dirección General de Educación Primaria de la Provincia de Córdoba, Boletín Oficial de la Provincia de Córdoba. (4 de noviembre de 2016). Extraída de https://boletinoficial.cba.gov.ar/wpcontent/4p96humuzp/2016/11/1 Secc 04112016.pdf

Mogollón de González, A. (2006). Funciones de la supervisión escolar en Venezuela. Aproximación a un modelo. Actualidades Investigativas en Educación. Vol.6, N³. Recuperado de https://dialnet.unirioja.es/servlet/ articulo?codigo $=2195357$

Morin, E. (1988). El método. Tomo III, Barcelona: Cátedra.

Morin, E. (1994). Introducción al Pensamiento Complejo. España: Gedisa.

Nicastro, S. (2019). Seminario Miradas sobre la figura del supervisor: garante, orientador e intérprete. ISEP. Córdoba. Argentina. Recuperado de http://isep-cba.edu.ar/web/2019/10/17/miradas-sobre-la-figuradel-supervisor-garante-orientador-e-interprete/

Pozner, P. (2007). El papel de la supervisión en la mejora de la calidad de la educación. In P. e. Pozner, Seminario Internacional Itinerante. Desarrollo de la gestión educativa en México: situación actual y perspectivas. (pp. 59-111). México: UPN.

Rivas, A y Furman, M. (2017). Seminario. Innovación y Justicia Educativa para Supervisores. CIPPEC. Laboratorio de Innovación y Justicia Educativa. Departamento de Desarrollos Multimedia, Ediciones Santillana S.A. Recuperado de http://edulab.cippec.org/edulabme/\#/lang/es/ pag/97f9e6804f4ff3f17fc81c09a8b3be07

Sarmiento, D. (1849). De la educación popular. Argentina: Imprenta de Julio Belin/Compañía.

Senestrari, N. (2015). La supervisión escolar y la gestión educativa en relación a la justicia educativa en el Sistema Educativo de Córdoba (Informe final de especialización) Universidad Nacional de Córdoba, Córdoba, Argentina. Recuperada de https://issuu.com/nestorgabrielsenestrari/docs/14-11reformulando_especializaci_n

Soler Fiérrez, E. (1994). La Inspección en las distintas concepciones y sistemas pedagógicos: características y funciones. (Tesis doctoral). Universidad Complutense. Madrid, España. Recuperada de https://eprints. ucm.es/3780/

Southwell, M. (2015). La Ley 1420 y la tarea de los Inspectores escolares. Historia de la Educación. Anuario. Vol.16, N¹. Recuperado de http://ppct.caicyt.gov.ar/index.php/anuario/article/view/7038

Suasnábar, C. (2017). Los ciclos de reforma educativa en América Latina: 1960, 1990 y 2000. Revista Española de Educación Comparada, №30, 112-135. Recuperado de file:///D:/Downloads/1987241312-2-PB.pdf 
Torres Hernández, A. (2007). La constitución de las prácticas de supervisión escolar en educación primaria dentro del orden institucional. (Tesis doctoral). Universidad Autónoma del Estado de Hidalgo, México. Recuperada de https://www.uaeh.edu.mx/nuestro_alumnado/icshu/doctorado/documentos/La\%20 constitucion\%20de\%20las\%20practicas.pdf 